\title{
Stress Imaging Utilization and Repeat Revascularization among Medicare Patients with High Risk Coronary Artery Disease
}

\author{
Joseph S. Rossi, MD ${ }^{a, b}$, Jerome J. Federspiel, AB $^{a, c}$, Daniel J. Crespin, MSPH ${ }^{\mathrm{e}}$, Timothy S. \\ Carey, MD ${ }^{a, d}$, Brett C. Sheridan, $\mathbf{M D}^{\mathrm{a}}$, and Sally C. Stearns, $\mathbf{P h D}^{\mathrm{c}, \mathrm{d}}$ \\ aUniversity of North Carolina School of Medicine, Chapel Hill, North Carolina \\ bSt. Vincent Medical Group, Indianapolis, Indiana \\ 'Department of Health Policy and Management, UNC Gillings School of Global Public Health, \\ Chapel Hill, North Carolina \\ ${ }^{d}$ Cecil G. Sheps Center for Health Services Research, Chapel Hill, North Carolina \\ eDepartment of Health Policy and Management, The University of Minnesota, Minneapolis, \\ Minnesota
}

\begin{abstract}
The optimal use of stress testing after coronary revascularization remains unclear, and overutilization of stress testing may increase rates of repeat revascularization. We analyzed the relationship at both the patient and regional level between the use of stress testing and repeat revascularization for a cohort of Medicare beneficiaries receiving revascularization within 30 days of an admission for symptomatic coronary artery disease (CAD).The sample consisted of 219,748 Medicare beneficiaries older than 65 years who received percutaneous coronary intervention (PCI) or cardiac bypass surgery (CABG) following hospital admission for symptomatic CAD in 20032004. Medicare claims data through 2008 identified the use of stress testing and repeat revascularization. Associations between the cumulative incidence of stress testing and repeat revascularization were analyzed using linear regressions. Within six years of initial revascularization, the cumulative incidence of events was 0.61 for stress testing and 0.23 for repeat revascularization. Most (53.1\%) repeat revascularizations were preceded by a stress test. Only $10.3 \%$ of repeat revascularization procedures were preceded by myocardial infarction. Fouryear cumulative incidence of repeat revascularization and stress testing varied between the Hospital Referral Regions represented by the sample, and the positive correlation between the rates by HRR accounted for only a small portion of the total HRR variation in revascularization rates. Stress testing is commonly performed among Medicare patients after initial revascularization, and the majority of repeat procedures are performed for stable CAD. Variation in stress testing patterns only explains a modest fraction of regional variation in repeat revascularization rates.
\end{abstract}

\section{Keywords}

Stress testing; Coronary revascularization; Medicare claims data

(C) 2012 Excerpta Medica, Inc. All rights reserved.

Corresponding Author: Joseph S. Rossi.

Publisher's Disclaimer: This is a PDF file of an unedited manuscript that has been accepted for publication. As a service to our customers we are providing this early version of the manuscript. The manuscript will undergo copyediting, typesetting, and review of the resulting proof before it is published in its final citable form. Please note that during the production process errors may be discovered which could affect the content, and all legal disclaimers that apply to the journal pertain. 
Although routine stress testing for patients with asymptomatic coronary artery disease (CAD) has been advocated for patients after PCI or CABG, current Appropriateness Criteria suggest that stress testing within 5 years of CABG or within 2 years of PCI is not appropriate in asymptomatic patients. ${ }^{1}$ In addition, current guidelines suggest that coronary revascularization should not be undertaken in asymptomatic patients unless there is evidence of high risk ischemia by non-invasive imaging. Despite these guidelines, geographic variation in use of stress testing by physicians may be associated with higher rates of repeat revascularization given the potentially discretionary nature of both decisions. To evaluate the contribution of regional variation in stress imaging utilization to the incidence of repeat revascularization, we conducted a retrospective cohort review of Medicare enrollees who underwent multivessel revascularization after admission for acute coronary syndrome.

\section{Methods}

The 2003 and 2004 Medicare Provider Analysis and Review (MedPAR) records were used to identify all fee-for-service Medicare beneficiaries aged 66 and older admitted for symptomatic CAD between January 1, 2003 and October 14, 2004, defined by the presence of International Classification of Diseases, Ninth Edition, Clinical Modification (ICD-9) diagnosis codes 410.xx, 411.1 and 413.9. Each patient's first CAD-related hospitalization during 2003 and 2004 was considered their index admission. For identified patients, we obtained MedPAR, Carrier, and Outpatient Medicare claims as well as the Medicare Denominator file for calendar years 2002 through 2008; as a result, up to six years of follow-up were available. Using claims from the 12 months preceding the index admission as well as the index admission itself, patients were excluded if they had a prior history of CAD-related admission, cardiac surgery, or percutaneous coronary intervention (PCI).

MedPAR and Carrier records were used to determine whether each identified patient received coronary revascularization during the 30 days following their index admission date based on ICD-9 procedure codes, Healthcare Common Procedure Coding System (HCPCS) codes, and Diagnosis-Related Groups (PCI: 36.01-36.07, 92980-92981, 92984, 9299592996, G0290-G0291, 516-518, 526-527, 555-558; CABG: 36.10-36.19, 33510-33523, 33530-33536, 106-107, 109, 547-550). Patients were included if their initial coronary revascularization procedure was a multivessel PCI or CABG. We also identified and included patients who received a staged PCI procedure by adding those patients who received a second PCI procedure within 30 days of their first, but for which the second PCI claim did not include an ACS-specific diagnosis code.

Carrier, Outpatient, and MedPAR records were next used to identify utilization of stress testing following revascularization using HCPCS and ICD-9 codes (stress testing with electrocardiogram (ECG): 93015-93018, 89.41-89.43; nuclear testing: 78460-78461, 78464-78466, 78468-78469, 78472-78473, 78481, 78483, 78491-78492, 78494, 89.44; echocardiography: 93350). ECG stress testing and nuclear stress testing claims occurring within 3 days of each other were considered a single, nuclear stress test; ECG stress testing and stress echocardiography testing occurring within 24 hours of each other were considered a single, echocardiography stress test. ${ }^{2}$ Because stress testing immediately subsequent to revascularization may be done to assess appropriateness for cardiac rehabilitation or to measure response to revascularization, stress tests were only included if they occurred more than 60 days from the date of the patient's first revascularization. Patients were included in the analyses if they survived 60 days subsequent to their initial revascularization procedure without repeat revascularization (with the exception of the staged PCI patients previously described) or readmission for myocardial infarction. 
The time to stress testing and time to repeat revascularization within 6 years of the initial revascularization were calculated using cumulative incidence techniques. Unadjusted cumulative incidence rates of repeat revascularization were examined both across the entire cohort and stratified by initial revascularization modality (CABG or PCI), with comparisons of cumulative incidence made using a non-parametric test of equality. ${ }^{3}$ Finally, patients were assigned to their Health Referral Region (HRR) from the Dartmouth Atlas based on the zip code on their index admission MedPAR record. Using observations aggregated at the HRR level, the correlation between the HRR-level cumulative incidence of repeat revascularization and stress testing was assessed using simple linear regression models weighted based on the number of cohort members in the HRR. The correlation between HRR-level incidence of stress testing, and the proportion of patients receiving a stress test who underwent repeat revascularization within 60 days of their stress test (the "test yield") was also compared using weighted linear regression.

Inferential statistics were considered significant at a two-sided alpha level of 0.05. All calculations were conducted in Stata/MP 12.0 (Statacorp, College Station, TX) except for competing risk calculations, which were performed in the R 2.12.1 (R Foundation, Vienna, Austria) using the cmprsk package. The University of North Carolina Public Health-Nursing Institutional Review Board reviewed and approved the study protocol and a waiver of informed consent.

\section{Results}

In total 86,132 patients met inclusion criteria, of which 54,124 were treated initially with CABG and 32,008 with PCI. Patients were followed for a mean of 1,639 days after revascularization (standard deviation 508 days). Baseline characteristics are reported in Table 1.

The cumulative incidence of having at least one repeat coronary revascularization procedure more than 60 days after the initial ACS-related revascularization was 0.17 . The cumulative incidence of repeat revascularization was higher (0.28) among PCI patients at 6 years of follow-up compared to 0.10 among CABG patients (Figure 1A; $p<0.001$ ). Coded indications for repeat revascularization were similar, regardless of the patient's initial revascularization modality (Table 2 ).

Using patient observations aggregated to the HRR, the cumulative incidence at 48 months after initial revascularization varied for both stress testing (median 0.55 , interquartile range [0.48 - 0.62]) and repeat revascularization (median 0.13, interquartile range [0.11 - 0.16]) (Figures 2A and 2B). Simple linear regressions showed that the proportion of patients in an HRR whose stress test was followed by a repeat revascularization with 60 days declined as the cumulative incidence of testing increased. (The overall regression coefficient was -0.08 , $\mathrm{p}<0.001$, with coefficients of -0.09 and -0.06 for PCI and CABG, respectively; $p<0.001$ for both. Full regression results are available on request from the authors.) Using linear regression weighted for the number of patients in each HRR, the HRR-level cumulative incidence of stress testing and repeat revascularization were positively and significantly associated at both 24 and 48 months following the initial revascularization, though the magnitude of the association was very modest (Table 3). For example, an increase in the cumulative incidence of stress testing of 0.1 at 48 months (roughly a $20 \%$ increase at the median) was only associated with an increase in the cumulative incidence or repeat revascularization of 0.0058 at 48 months (roughly only a $5 \%$ increase at the median). Furthermore, most of the association was attributable to $\mathrm{CABG}$ recipients, as stratification by initial revascularization modality showed that the association remained statistically significant for $\mathrm{CABG}$, but not for PCI. 


\section{Discussion}

Despite the high risk features of this population (Medicare patients with symptomatic coronary artery disease receiving multivessel revascularization), only $10.3 \%$ of repeat revascularization procedures were performed for patients presenting with myocardial infarction. The majority of procedures were performed for symptoms of angina or following a stress imaging study. These findings suggest that a large percentage repeat revascularization procedures are dependent on the evaluation of patients with stable coronary artery disease.

Higher rates of stress testing may contribute to higher rates of repeat revascularization among patients with stable symptoms. The unadjusted rates of repeat revascularization were particularly high among patients referred for PCI after initial presentation with acute coronary syndromes. However, the relative weakness of the regional association between stress imaging and repeat revascularization suggests that multiple factors are likely to contribute to repeat revascularization rates, including the mode of initial revascularization (PCI or CABG). We presented unadjusted comparisons because of the inability in this observational dataset to adjust for the many important but unobserved clinical factors that contribute to treatment decisions. The findings, however, are consistent with previous studies suggesting that $\mathrm{CABG}$ provides improved freedom from repeat revascularization procedures.

As the rate of stress testing increases, there is a decrease in the proportion of patients undergoing repeat revascularization (regression coefficient $-0.08, \mathrm{p}<0.001$ ). This result reflects a decrease in the yield of stress testing among regions with the highest testing rates. Shah et al. recently reported higher rates of stress testing after revascularization among patients whose physicians billed for both technical and professional fees for the procedure. ${ }^{4}$ The presence of physician-owned imaging equipment may have contributed to the variation in testing rates noted in this analysis.

Although we found variation in the utilization of both stress imaging and repeat coronary revascularization, the correlation of these two events was very modest. Unfortunately, sociodemographic and clinical data are not captured completely in Medicare claims. For instance, we were unable to determine the frequency of smoking within our cohort, and data regarding the location and severity of coronary lesions were also not available. In addition, variations in socioeconomic variables that may affect access to diagnostic testing are not accounted for in this analysis. Variation in unobserved procedural factors such as the use of arterial bypass conduits or the use of drug eluting stents may also affect revascularization rates. Although the use of Hospital Referral Regions was chosen in order to group the cohort according to geographic relevance to a tertiary care center, there is likely to be significant variation in practice patterns among hospitals and physicians within each region, and provider-level variation in stress testing rates was not explored.

The description of regional variation in practice patterns is important, because greater variation implies lack of a uniform standard of care. The utilization of stress imaging studies is largely dependent on provider-level practice patterns, and higher rates of testing do not appear to strongly predict higher rates of repeat revascularization, and therefore it must be considered that many of these tests are not necessary. Due to concerns regarding the utilization of diagnostic testing, the American College of Cardiology Foundation (ACCF) has released Appropriateness Criteria to serve as a guide for clinicians when determining the utility of these testing modalities for specific patient complaints and conditions. ${ }^{1,5}$ While the use of stress testing has decreased recently, ${ }^{6}$ the rate could increase in the future since the Centers for Medicare \& Medicaid Services has initiated a program to conduct audits for 
appropriateness of procedures including revascularization (www.cms.gov/apps/media/press/ factsheet.asp?Counter=4170). Although additional analysis is needed to evaluate the ongoing utilization of stress imaging studies in the Medicare population, our findings suggest that administrative data provide important insights into practice patterns, use of stress testing, and clinical outcomes.

\section{Acknowledgments}

Funding: National Institute on Aging (R01 AG025801), National Institute of General Medical Sciences (T32 GM008719), National Heart, Lung, and Blood Institute (F30 HL110483), all Bethesda, MD; Agency for Healthcare Research and Quality (T32 HS000032), Rockville, MD.

\section{References}

1. Douglas PS, Khandheria B, Stainback RF, Weissman NJ, Peterson ED, Hendel RC, Blaivas M, Des Prez RD, Gillam LD, Golash T, Hiratzka LF, Kussmaul WG, Labovitz AJ, Lindenfeld J, Masoudi FA, Mayo PH, Porembka D, Spertus JA, Wann LS, Wiegers SE, Brindis RG, Patel MR, Wolk MJ, Allen JM. ACCF/ASE/ACEP/AHA/ASNC/SCAI/SCCT/SCMR 2008 appropriateness criteria for stress echocardiography: A report of the aAmerican College of Cardiology Foundation Appropriateness Criteria Task Force, American Society of Echocardiography, American College of Emergency Physicians, American Heart Association, American Society of Nuclear Cardiology, Society for Cardiovascular Angiography and Interventions, Society of Cardiovascular Computed Tomography, and Society for Cardiovascular Magnetic Resonance endorsed by the Heart Rhythm Society and the Society of Critical Care Medicine. J Am Coll Cardiol. 2008; 51:1127-1147. [PubMed: 18342240]

2. Shah BR, Cowper PA, O'Brien SM, Jensen N, Drawz M, Patel MR, Douglas PS, Peterson ED. Patterns of cardiac stress testing after revascularization in community practice. J Am Coll Cardiol. 2010; 56:1328-1334. [PubMed: 20888523]

3. Gray RJ. A class of k-sample tests for comparing the cumulative incidence of a competing risk. Ann Stat. 1988; 16:1141-1154.

4. Shah BR, Cowper PA, O'Brien SM, Jensen N, Patel MR, Douglas PS, Peterson ED. Association between physician billing and cardiac stress testing patterns following coronary revascularization. JAMA. 2011; 306:1993-2000. [PubMed: 22068991]

5. Brindis RG, Douglas PS, Hendel RC, Peterson ED, Wolk MJ, Allen JM, Patel MR, Raskin IE, Bateman TM, Cerqueira MD, Gibbons RJ, Gillam LD, Gillespie JA, Iskandrian AE, Jerome SD, Krumholz HM, Messer JV, Spertus JA, Stowers SA. Accf/asnc appropriateness criteria for singlephoton emission computed tomography myocardial perfusion imaging (spect mpi): A report of the american college of cardiology foundation quality strategic directions committee appropriateness criteria working group and the american society of nuclear cardiology endorsed by the american heart association. J Am Coll Cardiol. 2005; 46:1587-1605. [PubMed: 16226194]

6. Shaw LJ, Marwick TH, Zoghbi WA, Hundley WG, Kramer CM, Achenbach S, Dilsizian V, Kern MJ, Chandrashekhar Y, Narula J. Why all the focus on cardiac imaging? JACC Cardiovasc imaging. 2010; 3:789-794. [PubMed: 20633864] 

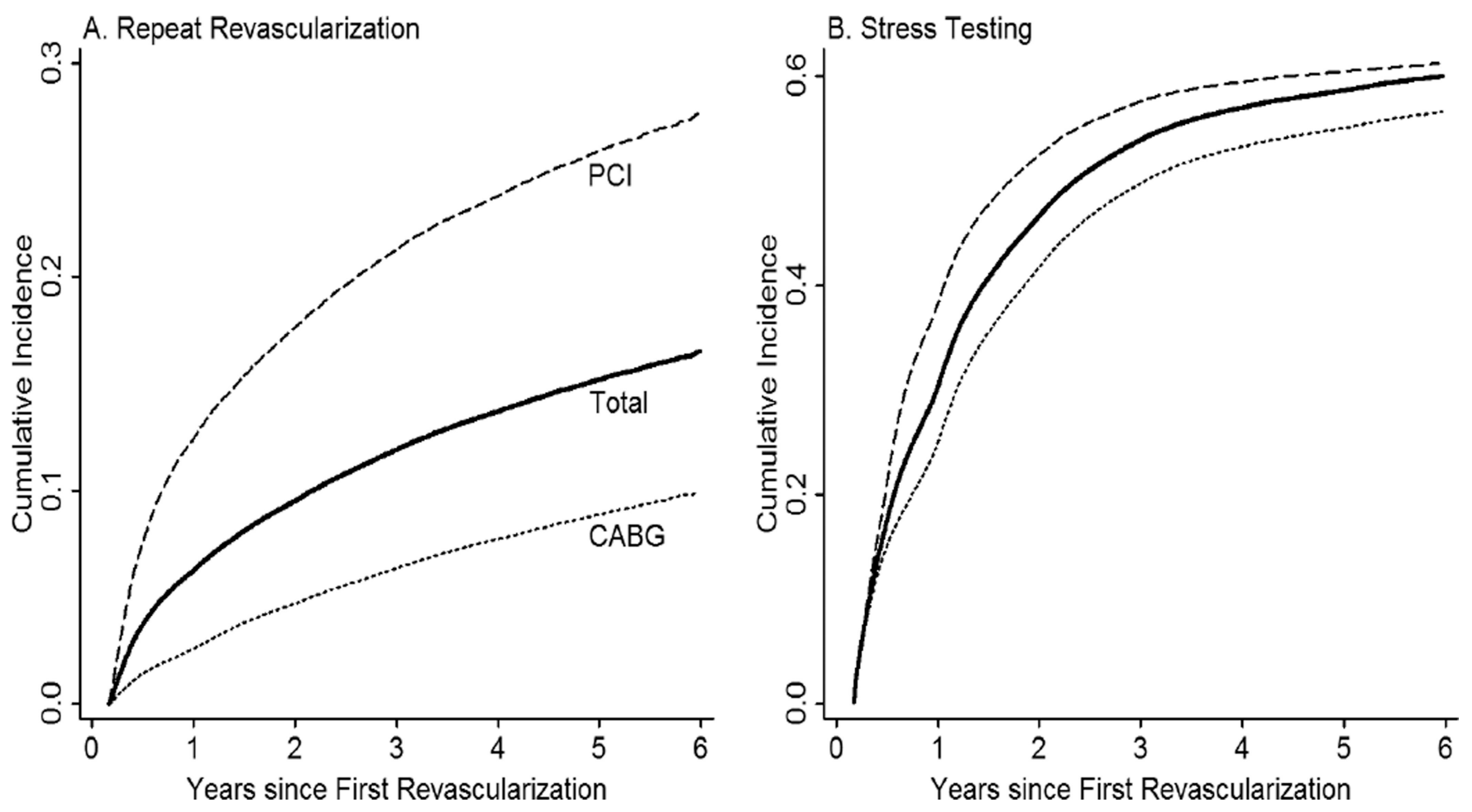

Figure 1.

Patient-level cumulative incidence of (A) repeat coronary revascularization and (B) stress testing, stratified by initial revascularization modality. 

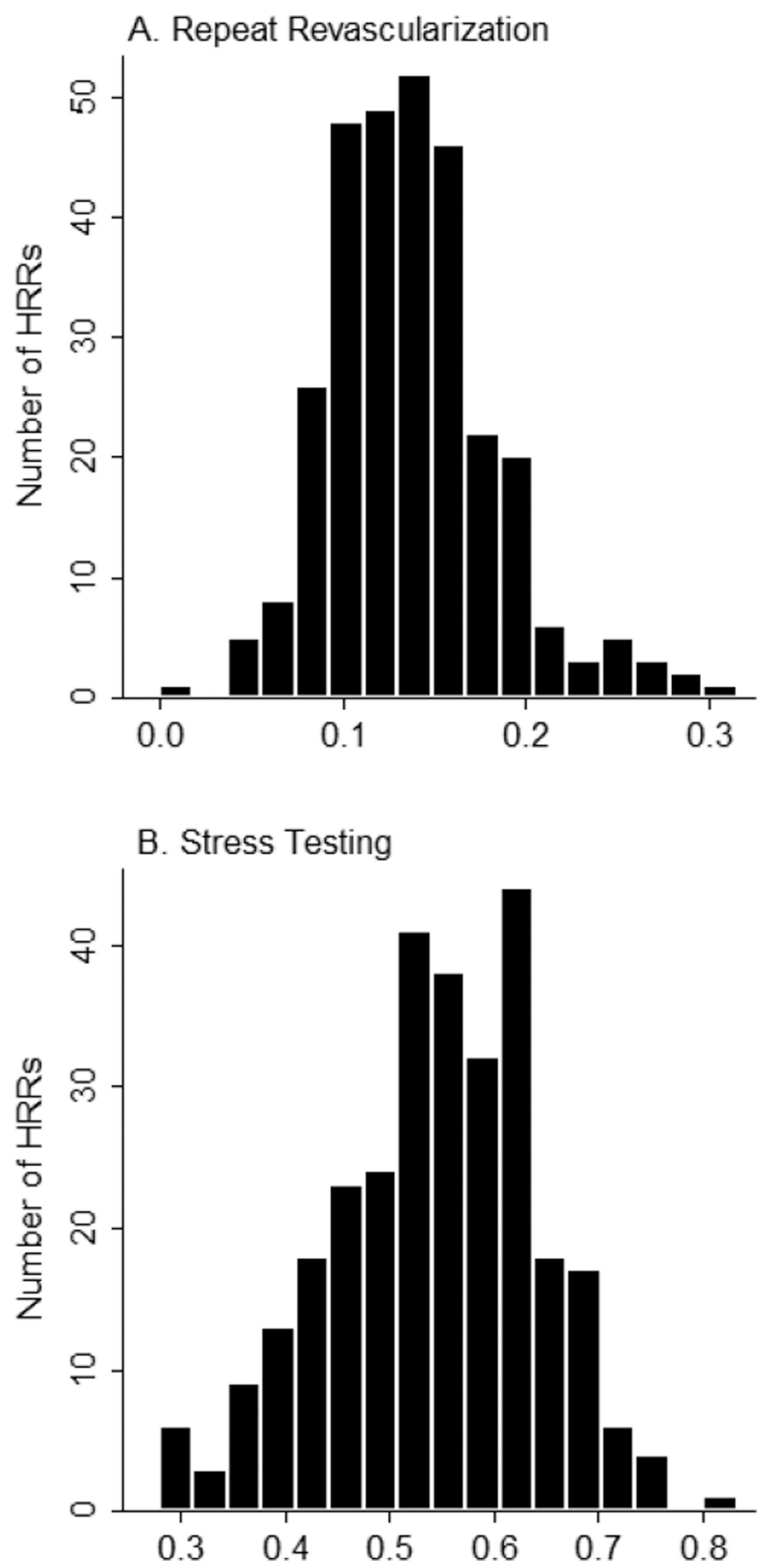

Figure 2.

Histogram of HRR-level cumulative incidence in (A) repeat coronary revascularization and (B) stress testing, both at 48 months after initial revascularization. 


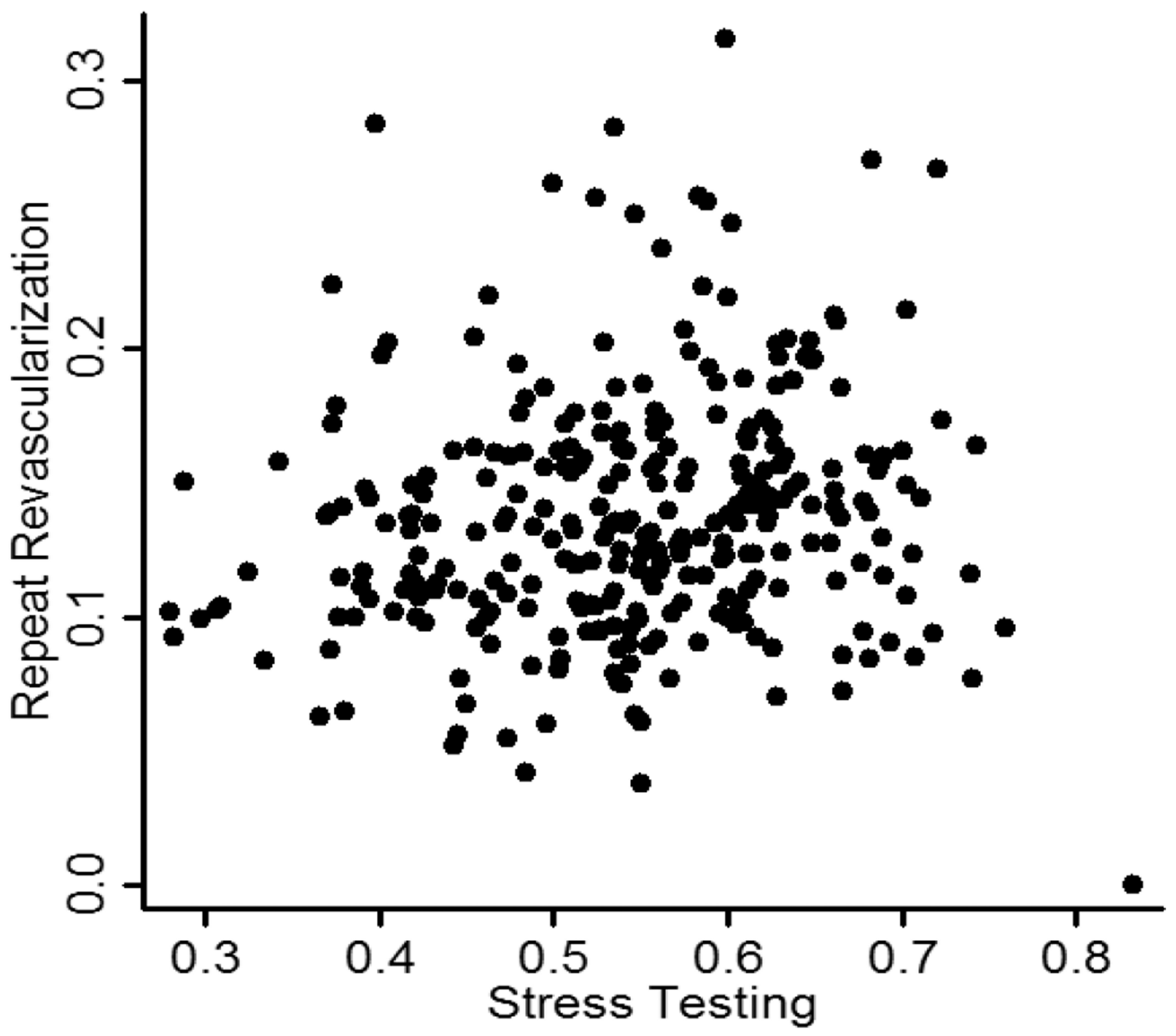

Figure 3.

Scatter plot illustrating correlation between HRR-level cumulative incidence of repeat revascularization and stress testing 


\section{Table 1}

Baseline Patient Characteristics Prior to Initial Acute Coronary Syndrome-Related Revascularization

\begin{tabular}{lcccc}
\hline Variable & $\begin{array}{c}\text { All } \\
(\mathbf{n = 8 6 , 1 3 2})\end{array}$ & $\begin{array}{c}\text { PCI } \\
(\mathbf{n = 3 2 , 0 0 8})\end{array}$ & $\begin{array}{c}\text { CABG } \\
(\mathbf{n = 5 4 , 1 2 4})\end{array}$ & p-value \\
\hline Mean Age (years) & 75.0 & 75.8 & 74.5 & $<0.001$ \\
Men & $60.3 \%$ & $53.6 \%$ & $64.2 \%$ & $<0.001$ \\
Diabetes Mellitus & $25.7 \%$ & $24.9 \%$ & $26.3 \%$ & $<0.001$ \\
Hypertension, with complications & $50.3 \%$ & $52.1 \%$ & $49.3 \%$ & $<0.001$ \\
Heart Failure & $6.4 \%$ & $8.4 \%$ & $5.3 \%$ & $<0.001$ \\
Prior Stroke & $7.2 \%$ & $6.9 \%$ & $7.4 \%$ & 0.03 \\
Chronic Kidney Disease/renal failure & $1.6 \%$ & $1.8 \%$ & $1.5 \%$ & 0.002 \\
Chronic Obstructive Pulmonary Disease & $10.2 \%$ & $12.2 \%$ & $9.0 \%$ & $<0.001$ \\
STEMI at presentation & $19.0 \%$ & $21.9 \%$ & $17.2 \%$ & $<0.001$ \\
\hline
\end{tabular}

$\mathrm{PCI}=$ percutaneous coronary intervention, $\mathrm{CABG}=$ coronary artery bypass grafting, $\mathrm{STEMI}=$ ST-Elevation Myocardial Infarction . 


\section{Table 2}

Coded indications for repeat revascularization, and percentage of patients whose repeat revascularization was preceded by a stress test, stratified by initial revascularization strategy.

\begin{tabular}{lcccc}
\hline $\begin{array}{l}\text { Diagnosis } \\
\text { ICD-9 Codes }\end{array}$ & $\begin{array}{c}\text { All } \\
(\mathbf{n = 1 3 , 2 9 2})\end{array}$ & $\begin{array}{c}\text { PCI } \\
(\mathbf{n = 8 , 3 6 0 )}\end{array}$ & $\begin{array}{c}\text { CABG } \\
(\mathbf{n = 4 , 9 3 2})\end{array}$ & p-value \\
\hline Angina 411.1, 413.x & $37.7 \%$ & $38.1 \%$ & $37.0 \%$ & 0.19 \\
Other Chest pain 786.50, 786.51, 786.59 & $10.5 \%$ & $10.6 \%$ & $10.4 \%$ & 0.69 \\
AMI 410.x1 & $10.3 \%$ & $10.6 \%$ & $9.8 \%$ & 0.15 \\
Preceded by stress test within 60 days of revascularization & $43.2 \%$ & $41.1 \%$ & $46.7 \%$ & $<0.001$ \\
\hline
\end{tabular}

$\mathrm{PCI}=$ percutaneous coronary intervention, $\mathrm{CABG}=$ coronary artery bypass grafting, $\mathrm{AMI}=$ acute myocardial infarction . 


\section{Table 3}

Association between stress testing and repeat revascularization by Hospital Referral Region

\begin{tabular}{lccc}
$\begin{array}{l}\text { Variable } \\
\text { 24 Months Post Revascularization }\end{array}$ & Regression Coefficient (CI) & P-value \\
\hline Overall & 297 & $0.048(0.012,0.084)$ & 0.01 \\
Percutaneous Coronary Intervention & 297 & $0.005(-0.057,0.067)$ & 0.87 \\
Coronary Bypass Surgery & 297 & $0.033(0.009,0.056)$ & 0.01 \\
$\mathbf{4 8}$ Months Post Revascularization & & & \\
\hline Overall & 297 & $0.058(0.010,0.107)$ & 0.02 \\
Percutaneous Coronary Intervention & 297 & $0.002(-0.077,0.081)$ & 0.95 \\
Coronary Bypass Surgery & 297 & $0.041(0.006,0.76)$ & 0.02 \\
\hline
\end{tabular}

